\title{
Temperature dependence of magnetic anisotropy of Ga-substituted cobalt ferrite
}

\author{
N. Ranvah, ${ }^{1, a)}$ Y. Melikhov, ${ }^{1}$ D. C. Jiles, ${ }^{1}$ J. E. Snyder, ${ }^{1}$ A. J. Moses, ${ }^{1}$ P. I. Williams, ${ }^{1}$ and \\ S. H. Song ${ }^{2}$ \\ ${ }^{1}$ Wolfson Centre for Magnetics, Cardiff University, Cardiff CF24 3AA, United Kingdom \\ ${ }^{2}$ Materials Science and Engineering Department, Iowa State University, Ames, Iowa 50011, USA
}

(Presented on 6 November 2007; received 5 October 2007; accepted 23 October 2007; published online 14 February 2008)

\begin{abstract}
The temperature dependence of magnetization, magnetic anisotropy, and coercive field of gallium-substituted cobalt ferrite was investigated for a series of compositions of $\mathrm{CoGa}_{x} \mathrm{Fe}_{2-x} \mathrm{O}_{4}$ $(0 \leqslant x \leqslant 0.8)$. Hysteresis loops were measured for each sample over the range of $-5 \mathrm{~T} \leqslant \mu_{0} H$ $\leqslant 5 \mathrm{~T}$ for selected temperatures between 10 and $400 \mathrm{~K}$. The magnetization at $5 \mathrm{~T}$ and low temperatures was found to increase for the lower Ga contents $(x=0.2$ and 0.4$)$ compared to pure $\mathrm{CoFe}_{2} \mathrm{O}_{4}$, indicating that at least initially, $\mathrm{Ga}^{3+}$ substitutes predominantly into the tetrahedral sites of the spinel structure. The high field regions of these loops were modeled using the law of approach to saturation, which represents the rotational process, together with an additional linear forced magnetization term. The first order cubic magnetocrystalline anisotropy coefficient $K_{1}$ was calculated from curve fitting to these data. It was found that $K_{1}$ decreased with increasing Ga content at all temperatures. Both anisotropy and coercivity increased substantially as temperature decreased. Below $150 \mathrm{~K}$, for certain compositions $(x=0,0.2,0.4)$, the maximum applied field of $\mu_{0} H=5 \mathrm{~T}$ was less than the anisotropy field and, therefore, insufficient to saturate the magnetization. In these cases, the use of the law of approach method can lead to calculated values of $K_{1}$ which are lower than the correct value. (C) 2008 American Institute of Physics. [DOI: 10.1063/1.2832503]
\end{abstract}

\section{INTRODUCTION}

There has been much interest recently in the properties of magnetoelastic cobalt ferrite and cobalt ferrite composites. ${ }^{1-4}$ These materials exhibit high levels of magnetostriction $\lambda$ and high sensitivity to field $d \lambda / d H$, which make them candidate materials for stress and torque sensor, and actuator applications. Substitution of a cation $M$ for $\mathrm{Fe}$ in $\mathrm{Co} M_{x} \mathrm{Fe}_{2-x} \mathrm{O}_{4}$ reduces the Curie temperature and improves magnetic and magnetoelastic properties in the cases of $M$ $=\mathrm{Mn}$ or $\mathrm{Cr}^{3,4}$ To fully control the magnetomechanical response of these materials, more needs to be known about their magnetic properties and their temperature dependences, especially the magnetic anisotropy. In the present work, the effect of gallium substitution on the temperature dependence of magnetization, magnetocrystalline anisotropy, and coercivity of $\mathrm{CoGa}_{x} \mathrm{Fe}_{2-x} \mathrm{O}_{4}(0 \leqslant x \leqslant 0.8)$ was investigated over the temperature range of $10-400 \mathrm{~K}$.

\section{EXPERIMENT AND RESULTS}

A series of gallium-substituted cobalt ferrite samples was prepared by standard ceramic techniques ${ }^{1,2}$ with a final sintering at a temperature of $1350{ }^{\circ} \mathrm{C}$ for $24 \mathrm{~h}$, followed by furnace cooling to room temperature. Energy dispersive x-ray spectroscopy (EDS) was used to determine the actual final compositions of the samples, which were found to be close to the target compositions. Table I shows the comparison of target and actual compositions.

Dependence of technical saturation magnetization on

${ }^{a)}$ Electronic mail: ranvahn@cardiff.ac.uk. temperature was measured using a superconducting quantum interference device (SQUID) magnetometer under an applied field of $5 \mathrm{~T}$, as shown in Fig. 1. It was observed that magnetization monotonically increased as temperature decreased from 400 to $160 \mathrm{~K}$ for all samples. Below $160 \mathrm{~K}$, magnetization increased slowly with decreasing temperature for most samples but decreased for pure $\mathrm{CoFe}_{2} \mathrm{O}_{4}$ (from $160 \mathrm{~K}$ ) and $\mathrm{CoGa}_{0.2} \mathrm{Fe}_{1.8} \mathrm{O}_{4}$ (from $100 \mathrm{~K}$ ). This apparent decrease can be explained by the presence of high anisotropy fields at low temperatures for these samples, which prevented complete saturation of magnetization under the field strengths used.

Symmetric magnetic hysteresis loops were measured over temperatures ranging from 10 to $400 \mathrm{~K}$ using a SQUID magnetometer, with a maximum applied field of $5 \mathrm{~T}$. An example of the temperature dependence of the first quadrant of the hysteresis curves is given in Fig. 2 for $\mathrm{CoGa}_{0.4} \mathrm{Fe}_{1.6} \mathrm{O}_{4}$. The coercive fields for each sample at different temperatures are shown in Fig. 3.

For the calculation of anisotropy, it was assumed that

TABLE I. Comparison of target and actual compositions in $\mathrm{CoGa}_{x} \mathrm{Fe}_{2-x} \mathrm{O}_{4}$.

\begin{tabular}{lccc}
\hline \hline \multirow{2}{*}{$\begin{array}{c}\text { Target } \\
\text { composition }\end{array}$} & \multicolumn{3}{c}{ Composition by EDS } \\
\cline { 2 - 4 } & $\mathrm{Co}$ & $\mathrm{Fe}$ & $\mathrm{Ga}$ \\
\hline $\mathrm{CoFe}_{2} \mathrm{O}_{4}$ & 0.95 & 2.05 & $\ldots$ \\
$\mathrm{CoGa}_{0.2} \mathrm{Fe}_{1.8} \mathrm{O}_{4}$ & 1.00 & 1.81 & 0.19 \\
$\mathrm{CoGa}_{0.4} \mathrm{Fe}_{1.6} \mathrm{O}_{4}$ & 1.04 & 1.55 & 0.41 \\
$\mathrm{CoGa}_{0.6} \mathrm{Fe}_{1.4} \mathrm{O}_{4}$ & 0.98 & 1.33 & 0.69 \\
$\mathrm{CoGa}_{0.8} \mathrm{Fe}_{1.2} \mathrm{O}_{4}$ & 1.04 & 1.15 & 0.81 \\
\hline \hline
\end{tabular}




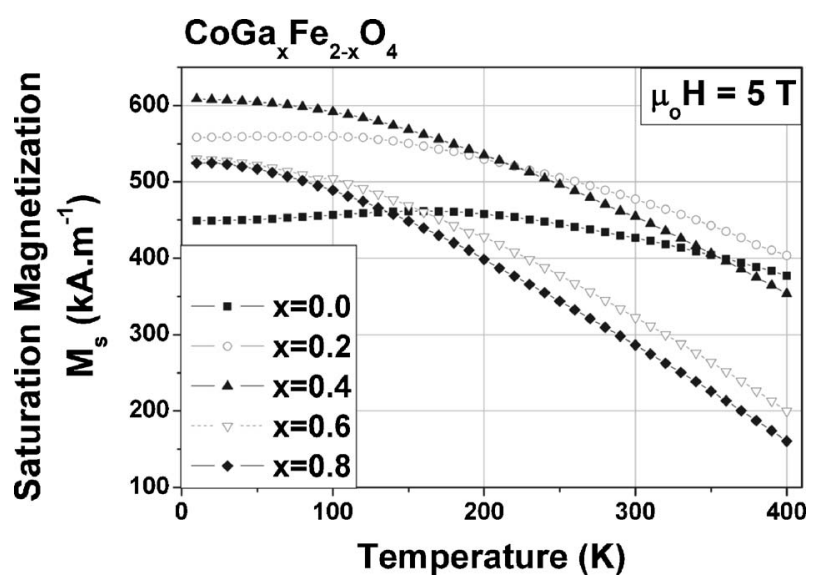

FIG. 1. Temperature dependence of magnetization of Ga-substituted cobalt ferrite at an applied field of $5 \mathrm{~T}$.

after the closing of the hysteresis loops, all the irreversible processes were finished. The high field regions represent the processes of rotation against anisotropy and at the higher temperatures, forced magnetization. According to the law of approach (LA) to saturation, ${ }^{5}$ the effect of these processes on magnetization, for $H_{\gg} H_{C}$, can be modeled by the equation

$$
M=M_{s}\left[1-\frac{8}{105} \frac{K_{1}^{2}}{\mu_{0}^{2} M_{s}^{2}} \frac{1}{H^{2}}\right]+\kappa H,
$$

where $M$ is magnetization, $M_{s}$ is saturation magnetization, $K_{1}$ is first order cubic anisotropy coefficient, $H$ is magnetic field, and $\kappa$ is the forced magnetization coefficient representing the linear increase in spontaneous magnetization at high applied field values. The constant 8/105 applies to first order cubic anisotropy of random polycrystalline samples.

The data from high field regimes were fitted to Eq. (1). To extract the most appropriate values of $K_{1}, M_{s}$, and $\kappa$, only data at applied fields above $1 \mathrm{~T}$ were used to fit to the LA for temperatures above $150 \mathrm{~K}$. For temperatures below $150 \mathrm{~K}$, only data at applied fields higher than $2.5 \mathrm{~T}$ were fitted to the LA. The values of anisotropy coefficient $K_{1}$ computed using this procedure are shown in Fig. 4. For low Ga content $(x$ $=0,0.2,0.4)$ and low temperatures $(T=100,50$, and $10 \mathrm{~K})$, it was observed that the value of kappa, calculated directly from the fits, increased with decreasing temperature, which is

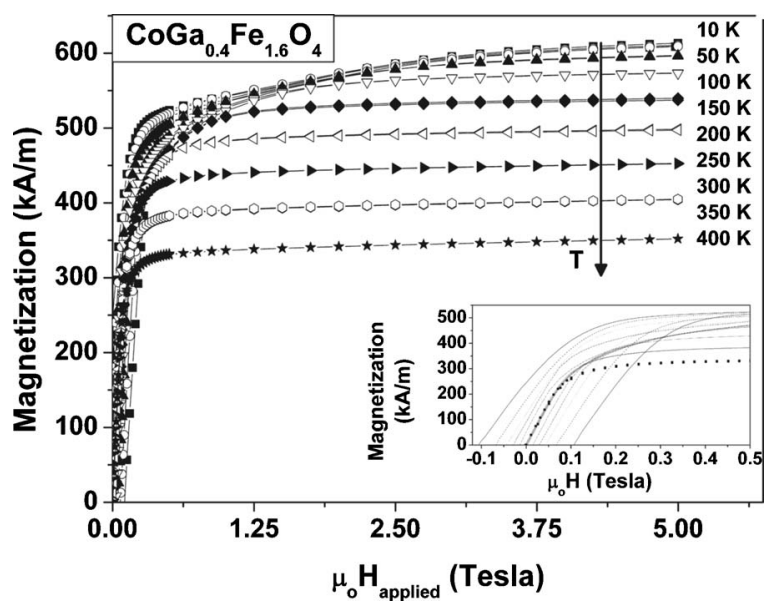

FIG. 2. First quadrant of hysteresis loops for $\mathrm{CoGa}_{0.4} \mathrm{Fe}_{1.6} \mathrm{O}_{4}$. The inset shows the low field regions of hysteresis loops used to calculate coercivity.

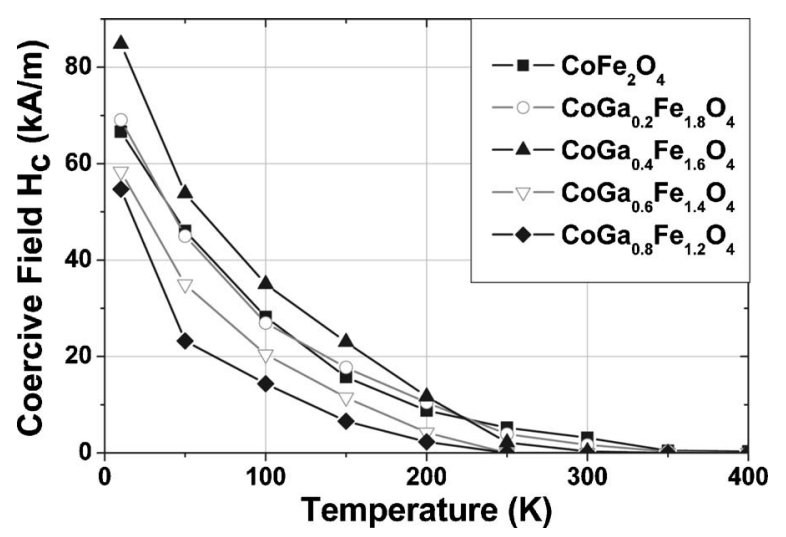

FIG. 3. Temperature dependence of coercive fields for $\mathrm{CoGa}_{x} \mathrm{Fe}_{2-x} \mathrm{O}_{4}$.

an artefact. In these cases, the estimated anisotropy fields were greater than the maximum applied field, thus indicating that anisotropy dominated over forced magnetization (see Conclusion and Refs. 3 and 4). Forced magnetization was assumed to be negligible at these temperatures and the experimental data were refitted to the LA with $\kappa=0$ for these points so that $M_{s}$ and $K_{1}$ were the only fitting parameters for these calculations. These points are connected with a dotted line in Fig. 4. The values of $M_{s}$ obtained by fitting the experimental data to Eq. (1) were in agreement with the magnetization measured at an applied field of $5 \mathrm{~T}$, except for the samples with $x \leqslant 0.2$ for temperatures below $150 \mathrm{~K}$. In these cases, the values obtained from the calculations were slightly above the measured values, which is consistent with the idea that the anisotropy field is greater than the maximum applied field.

\section{DISCUSSION}

Starting from $400 \mathrm{~K}$, the anisotropy coefficient $K_{1}$ is observed to increase with decreasing temperature for all compositions, with the region of steepest increase coming at progressively lower temperatures for increasing Ga content until $x=0.6$, then the $x=0.8$ sample was essentially the same as the $x=0.6$ sample. However, for the low Ga contents $(x=0,0.2$, or 0.4) and lower temperatures, $K_{1}$ calculated from the LA fits was observed to peak and decrease or level off. This

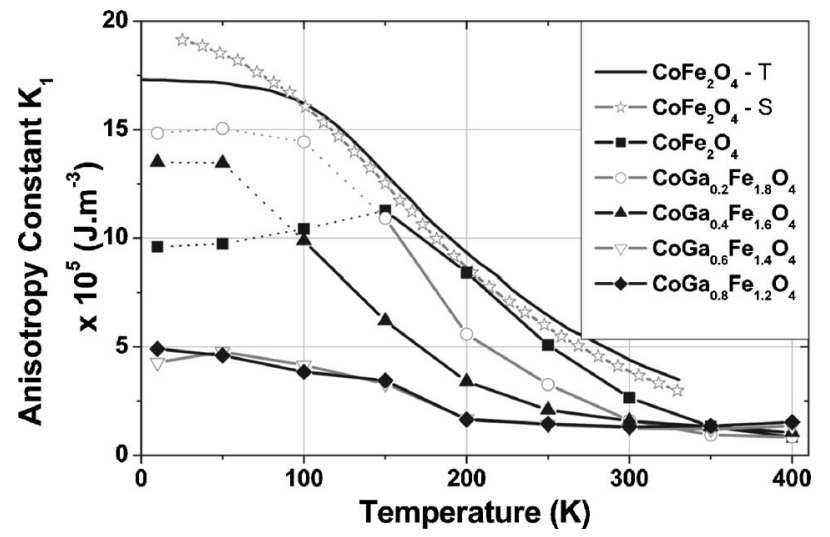

FIG. 4. Temperature dependence of cubic anisotropy constant $K_{1}$, calculated by fitting experimental data to $\mathrm{LA}$, for $\mathrm{CoGa}_{x} \mathrm{Fe}_{2-x} \mathrm{O}_{4}$. Experimental data (Ref. 8) marked as $\mathrm{CoFe}_{2} \mathrm{O}_{4}-\mathrm{S}$ and theoretically calculated data (Ref. 9) marked as $\mathrm{CoFe}_{2} \mathrm{O}_{4}-\mathrm{T}$ are presented for comparison. 
apparent decrease was because the anisotropy fields of these samples reach such high levels at low temperatures that the applied fields of up to $\mu_{0} H=5 \mathrm{~T}$ (maximum available) were unable to saturate the magnetization.

Estimation of the anisotropy fields for these samples supports this idea. For a cubic crystal with the $\langle 100\rangle$ easy directions such as cobalt ferrite, $H_{K}=2 K_{1} / \mu_{0} M_{s} .{ }^{6}$ The anisotropy field for pure cobalt ferrite at $150 \mathrm{~K}$ was estimated to be $4.8 \mathrm{~T}$, and it should increase further at lower temperatures. Similarly, the estimated anisotropy field for the specimen with $x=0.2$ at $100 \mathrm{~K}$ was $5.16 \mathrm{~T}$. These ideas are also supported by the magnetization data for low Ga contents and low temperatures. For example, for pure cobalt ferrite $(x=0)$, magnetization has a maximum at $160 \mathrm{~K}$ for pure cobalt ferrite and for the gallium-substituted sample with $x$ $=0.2$, magnetization has a maximum at $100 \mathrm{~K}$ (see Fig. 1). Therefore, the anisotropy prevents a complete approach to saturation in these cases and, hence, the values of $K_{1}$ calculated from fitting the experimental data to the LA equation are unreliable.

The LA gives the absolute value of the cubic anisotropy coefficient $K_{1}$. To second order, the numerical expression is the same for cubic anisotropy with the $\langle 100\rangle$ and $\langle 111\rangle$ easy axes. Pure cobalt ferrite has been reported to have the $\langle 100\rangle$ easy axis directions, with $K_{1}$ values that are positive and range from $2.1 \times 10^{5}$ to $3.9 \times 10^{5} \mathrm{~J} / \mathrm{m}^{3}$ at $300 \mathrm{~K}$, with variation caused by heat treatment and stoichiometry of the material. ${ }^{7}$ The value of $K_{1}$ for pure cobalt ferrite at $300 \mathrm{~K}$, in the present study, is $2.66 \times 10^{5} \mathrm{~J} / \mathrm{m}^{3}$. This is consistent with the results of Shenker, ${ }^{8}$ who measured anisotropy for singlecrystal pure cobalt ferrite and also with the theoretical work of Tachiki for pure cobalt ferrite ${ }^{9}$ (see Fig. 4).

Despite the fact that the $5 \mathrm{~T}$ applied field cannot entirely saturate the lower Ga-content samples at the lower temperatures, magnetization at $10 \mathrm{~K}$ is clearly observed to increase for the $x=0.2$ and $x=0.4$ samples compared to pure $\mathrm{CoFe}_{2} \mathrm{O}_{4}$ (see Fig. 1). This indicates that initially, at least, the $\mathrm{Ga}^{3+}$ ions substitute into the tetrahedral sites in the inverse spinel structure. $\mathrm{Ga}^{3+}$ has ten $3 d$ electrons and, thus, no magnetic moment. The net moment of ferrimagnetic spinels is determined by the difference of the octahedral moments $M_{B}$ minus the tetrahedral moments $M_{A}$. Thus, the only way that substituting nonmagnetic ions can make the magnetization increase, is if they substitute onto the tetrahedral sites. It is not clear why the low temperature magnetization decreases for the higher $\mathrm{Ga}$ contents $(x=0.6$ and 0.8$)$. It could be due to the additional Ga substituting into the octahedral sites for these compositions, or to noncollinear spin arrangements brought on by the decreased tetrahedral-octahedral exchange coupling. This will require further investigation.

The assertion that for low concentrations, $\mathrm{Ga}^{3+}$ ions occupy tetrahedral sites, is further supported by recent Mössbauer spectroscopy data, which indicate that $\mathrm{Ga}^{3+}$ ions appear to favor the tetrahedral sites in $\mathrm{CoGa}_{x} \mathrm{Fe}_{2-x} \mathrm{O}_{4} \cdot{ }^{10} \mathrm{Mn}^{3+}$ and $\mathrm{Cr}^{3+}$, on the other hand, substitute into the octahedral sites and displace some of the $\mathrm{Co}^{2+}$ onto the tetrahedral sites. ${ }^{11,12}$ According to the one-ion anisotropy model, the high anisotropy of cobalt ferrite is primarily due to the presence of $\mathrm{Co}^{2+}$ ions on the octahedral sites of the spinel struc- ture (see, for example, Ref. 5). For low concentrations of $\mathrm{Ga}^{3+}(x=0.2$ and 0.4$)$, although $\mathrm{Ga}^{3+}$ ions go into tetrahedral sites, they still reduce the anisotropy. This is thought to be due to the reduction of the tetrahedral-octahedral exchange coupling. For the samples $x=0.6$ and 0.8 , however, the $K_{1}$ reduction could be caused by either of two mechanisms. Anisotropy could either decrease due to the additional Ga substituting into the octahedral sites for these compositions (and displacing $\mathrm{Co}^{2+}$ ions to tetrahedral sites), or due to further decrease of the tetrahedral-octahedral exchange coupling, which brings on noncollinear spin arrangements.

\section{CONCLUSION}

The effect of Ga substitution on the temperature dependence of the magnetic anisotropy, magnetization, and coercivity of polycrystalline cobalt ferrite $\mathrm{CoGa}_{x} \mathrm{Fe}_{2-x} \mathrm{O}_{4}(0 \leqslant x$ $\leqslant 0.8$ ) was investigated over the temperature range of $10-400 \mathrm{~K}$. The first order cubic anisotropy coefficient $K_{1}$ was estimated by fitting the high field region of the hysteresis loops to the law of approach to saturation. It was found that anisotropy of all Ga-substituted cobalt ferrite samples increased with decreasing temperature. It was also found that the anisotropy of gallium-substituted cobalt ferrite decreased with increasing gallium content at all temperatures. It was observed that the magnetization at $10 \mathrm{~K}$ increased for lower Ga contents ( $x=0.2$ and 0.4 ) compared to pure cobalt ferrite. The results indicate that $\mathrm{Ga}$ ions preferentially substitute into the tetrahedral sites. It was concluded that the anisotropy of $\mathrm{CoGa}_{x} \mathrm{Fe}_{2-x} \mathrm{O}_{4}$ for low Ga content $(x=0,0.2$, and 0.4$)$ was so high at low temperatures that it prevented complete approach to saturation, similar to what had been observed with $\mathrm{Mn}$ or Cr substitution. ${ }^{3,4}$ Thus, it is not possible to reliably calculate the values of $K_{1}$ at these low temperatures using the law of approach method.

\section{ACKNOWLEDGMENTS}

This research was supported by the U.K. Engineering and Physical Sciences Research Council under Grant No. EP/D057094 and by the U.S. National Science Foundation under Grant No. DMR-0402716.

\footnotetext{
${ }^{1}$ Y. Chen, J. E. Snyder, C. R. Schwichtenberg, K. W. Callum, and D. C. Jiles, IEEE Trans. Magn. 35, 3652 (1999).

${ }^{2}$ J. A. Paulsen, A. P. Ring, C. C. H. Lo, J. E. Snyder, and D. C. Jiles, J. Appl. Phys. 97, 044502 (2005).

${ }^{3}$ Y. Melikhov, J. E. Snyder, C. C. H. Lo, P. N. Matlage, S. H. Song, K. W. Dennis, and D. C. Jiles, IEEE Trans. Magn. 42, 2861 (2006).

${ }^{4}$ Y. Melikhov, J. E. Snyder, C. C. H. Lo, A. P. Ring, J. A. Paulsen, K. W. Dennis, and D. C. Jiles, J. Appl. Phys. 99, 08R102 (2006).

${ }^{5}$ S. Chikazumi, Physics of Ferromagnetism, 2nd ed. (Oxford University Press, Oxford, 1997), pp. 503-508.

${ }^{6}$ B. D. Cullity, Introduction to Magnetic Materials (Addison-Wesley, Reading, MA, 1972), p. 233.

${ }^{7}$ J. Smit, Ferrites (Wiley, New York, 1959), p. 163.

${ }^{8}$ H. Shenker, Phys. Rev. 107, 1246 (1957).

${ }^{9}$ M. Tachiki, Prog. Theor. Phys. 23, 1055 (1960).

${ }^{10}$ K. Krieble, M. Devlin, S. J. Lee, S. T. Aldini, and J. E. Snyder, "Investigation of $\mathrm{Ga}$ substitution in Cobalt-Ferrite $\left(\mathrm{CoGa}_{x} \mathrm{Fe}_{2-x} \mathrm{O}_{4}\right)$ using Mossbauer spectroscopy," J. Appl. Phys. (these proceedings), Abstract No. BH-15.

${ }^{11}$ K. Krieble, C. C. H. Lo, Y. Melikhov, and J. E. Snyder, J. Appl. Phys. 99, 08M812 (2006).

${ }^{12}$ K. Krieble et al., J. Appl. Phys. 97, 10F101 (2005).
} 